\title{
Impact of dual dispatch system implementation on response times and survival outcomes in out-of-hospital cardiac arrest in rural areas
}

\author{
Matej Strnad ${ }^{1,2,3, \star}$, Pia Jerot ${ }^{4}$, Vesna Borovnik Lesjak ${ }^{1}$
}

${ }^{1}$ Center for Emergency Medicine, Prehospital unit, Community healthcare center, 2000 Maribor, Slovenia

${ }^{2}$ Emergency department, University Clinical Centre Maribor, 2000 Maribor, Slovenia

${ }^{3}$ Department of Emergency Medicine, Medical faculty, University of Maribor, 2000 Maribor, Slovenia

${ }^{4}$ Community healthcare center, 2360

Radlje ob Dravi, Slovenia

*Correspondence

strnad.matej78@gmail.com

(Matej Strnad)

\begin{abstract}
Objectives: Dual dispatch early defibrillation in out-of-hospital cardiac arrest (OHCA) victims provided by firefighters in addition to Emergency medical services (EMS) has proven to increase rate of return of spontaneous circulation (ROSC) and thus survival in the metropolitan or suburban areas whereas the data in rural areas are scarce.

Methods: This was a retrospective observational cohort study of EMS resuscitated OHCA victims in regions with dual dispatch of volunteer firefighters as first responders (intervention group). Historical group was based on all OHCAs occurring in these regions before the implementation of first responders (EMS response only). Multivariate logistic regression with following variables: intervention, age, gender, witnessed status, bystander cardiopulmonary resuscitation (CPR), first rhythm and etiology were used to control for confounding factors affecting ROSC.

Results: A total of 312 OHCAs were included in the study (historical group, $n=115$ and intervention group, $n=197$ ). Median time to arrival of first help shortened significantly for all patients, patients with ROSC and patients with Cerebral Performance Category $1 / 2$ (CPC 1/2) in intervention vs historical group ( 8 vs $12 \mathrm{~min}, p<0.001 ; 7.5$ vs $11 \mathrm{~min}$, $p=0.002 ; 7$ vs $10 \mathrm{~min}, p=0.011$; respectively). The proportion of patients with ROSC, 30-day survival and CPC $1 / 2$ at hospital discharge remained unchanged in intervention vs historical group $(21 \%$ vs $23 \%, p=0.808 ; 7 \%$ vs $6 \%, p=0.914 ; 6 \%$ vs $3 \%, p=$ 0.442 ; respectively). The logistic regression model of adjustment confirms the absence of improvement in the ROSC rate after the implementation of first responders.

Conclusions: Introduction of a dual dispatch of local first responders in addition to EMS in cases of OHCA significantly shortened response times. However, reduced response times were not associated with better survival outcomes.
\end{abstract}

\section{Keywords}

Out-of-hospital cardiac arrest; First responder; Survival rate; Sudden cardiac death; Automated external defibrillator; Cardiopulmonary resuscitation; Firefighters

\section{Introduction}

Out-of-hospital cardiac arrest (OHCA) is one of the leading causes of death and annually affects $\sim 275,000$ people in Europe $[1,2]$. Bystander cardiopulmonary resuscitation (CPR) [3] and early defibrillation with automated external defibrillator (AED) are essential in improving survival of OHCA victims [4, 5]. Different strategies were implemented to support Emergency medical services (EMS) reduce the time interval to first shock. Public access defibrillation programs place AEDs in densely populated areas and in locations with many visitors, where AEDs can be used by bystanders [5-7]. Other programs include first responders with AEDs like police officers [8-10], firefighters [11-14], local lay rescuers $[15,16]$ or any combination thereof $[4,17,18]$ dispatched to a suspected OHCA victim as a part of organized EMS response. Dual dispatch early defibrillation in OHCA provided by firefighters increases survival and was studied predominantly in the metropolitan or suburban areas [11, 14, 17, 19], whereas in rural areas the data are scarce $[12,13]$. OHCA victims in rural areas benefit from local first responders due to shorter driving distance thus reducing the time interval to first shock and minimizing the risk that an initial shockable rhythm deteriorates to a non-shockable rhythm, which decreases the chance of survival [20-22]. A time difference of as little as 1 minute appears to significantly influence survival [17].

The aim of the present observational study was to determine the impact of volunteer firefighter interventions as local first responders on response times and survival outcomes in OHCA victims in rural areas. 


\section{Methods}

The regional ethics committee of University Medical Center Maribor approved the study and waived the requirement to obtain any informed consent (No. UKC-MB-KME-24/20, 02/010/03-003/01/20).

\subsection{Setting}

This study took place in the northeastern part of Slovenia. The study region included surrounding catchment rural areas with several smaller and bigger villages of the following EMS: Maribor, Ptuj, Velenje and Koroška region with the population of 138,281 covering $1504.6 \mathrm{~km}^{2}$ and average population density of 91.9 people $/ \mathrm{km}^{2}$. In this area, 63 local fire stations are equipped with AEDs.

\subsection{Emergency medical services (EMS)}

The EMS in Slovenia is provided by the Community healthcare centers with EMS stations located in the urban areas. The catchment area of a single EMS expands over several surrounding municipalities and thus the EMS provides its service not only in the urban/suburban but also in the rural areas with response times up to $40 \mathrm{~min}$. When a cardiac arrest is suspected, an ambulance with two paramedics and an emergency physician on board is dispatched.

\subsection{First responders}

From 2014 first responders (local voluntary firefighters) have been organized in the rural areas with the EMS response times of more than $10 \mathrm{~min}$. Median response time of EMS included in the study is $8 \mathrm{~min}$ in the urban/suburban areas. According to the Rules on Emergency Medical Service published in Official Journal of the Republic of Slovenia all first responders have to undergo a 10-hour training to gain the following knowledge and skills in basic life support (BLS): adult and children CPR with the use of an AED according to the European Resuscitation Council (ERC) guidelines, foreign body airway obstruction and control of the major isolated traumatic external bleeding. Initially, the ERC guidelines from 2010 were used for the training [23]. In the beginning of 2016, the training was adapted to the 2015 guidelines [24]. The algorithms of the AED were changed accordingly. After the training, the participants have to pass a written and a practical exam to obtain the first responder license. All first responders have to renew their license annually.

\subsection{Dual dispatch}

In Slovenia, Regional Emergency Notification Center receives all 112-emergency calls. In case of a medical emergency, the emergency call is forwarded to the Medical dispatch center. In cases of suspected cardiac arrests, the Medical dispatch center alerts the nearest available ambulance (EMS) with emergency physician on board first and then, as soon as possible, dispatches the closest available fire brigade with organized local first responders via the Regional Emergency Notification Center. The fire brigade dispatch should happen simultaneously with the EMS dispatch. The first responders to arrive at the scene are responsible for performing medical assessment. If the patient is unresponsive and not breathing, CPR is initiated and the AED is attached. When the EMS crew arrives, they take over the responsibility for the treatment, but the first responders are included in ongoing resuscitation as additional help.

\subsection{Study design}

This is a retrospective observational cohort study of the prospective gathered data in regions where a dual dispatch of first responders was implemented (intervention group). Enrollment of the patients in the intervention group depended on the time of the first responder implementation in each studied region. In EMS Maribor and EMS Koroška region the first responder system had been stepwise organized since May 2014 and was fully implemented in January 2015. Within the Northeastern Slovenia the system was incrementally rolled out to the other included regions with complete coverage from December 2017. Historical control group was based on all OHCAs occurring in these regions before the implementation of first responders (EMS response only). Comparison of all OHCA victims for an equivalent time period in both groups was made (see Appendix Fig. 2).

EMS protocols designed according to the Utstein style reporting for OHCA, first responder intervention reports, dispatch protocols of the Medical dispatch center and hospital medical reports for the study period were analyzed. Calculations of times and intervals were based on the above-mentioned reports.

\subsection{Study participants}

Eligible patients were all EMS resuscitated OHCAs in the rural areas included in the study (independent of witnessed status, cardiac rhythm and previous morbidity). The exclusion criteria were: age $\leq 18$ years, EMS crew-witnessed OHCAs, in-hospital arrests, patients with obvious signs of death, people with existing Do Not Attempt Resuscitation (DNAR) orders and patients where no CPR was initiated by the EMS.

\subsection{Endpoints}

The primary endpoints were: (1) number of OHCAs with the return of spontaneous circulation (ROSC), (2) 30-day survival and (3) neurological outcome. Secondary endpoints were: (1) first responder response times, (2) proportion of patients admitted alive to hospital and (3) proportion of patients with VF/VT as initial rhythm.

\subsection{Statistical analyses}

Baseline characteristics of the sample are presented with frequencies (means, standard deviations, number, and percentages). Comparisons between intervention and historical groups were analyzed with $t$-tests for age and Chi squared test for categorical data. Median time intervals and survival between groups were compared with Mann-Whitney $U$ test due to non-normal distribution. Multivariate logistic regression was used to control for confounding factors affecting ROSC between the intervention and the historical 
Intervention

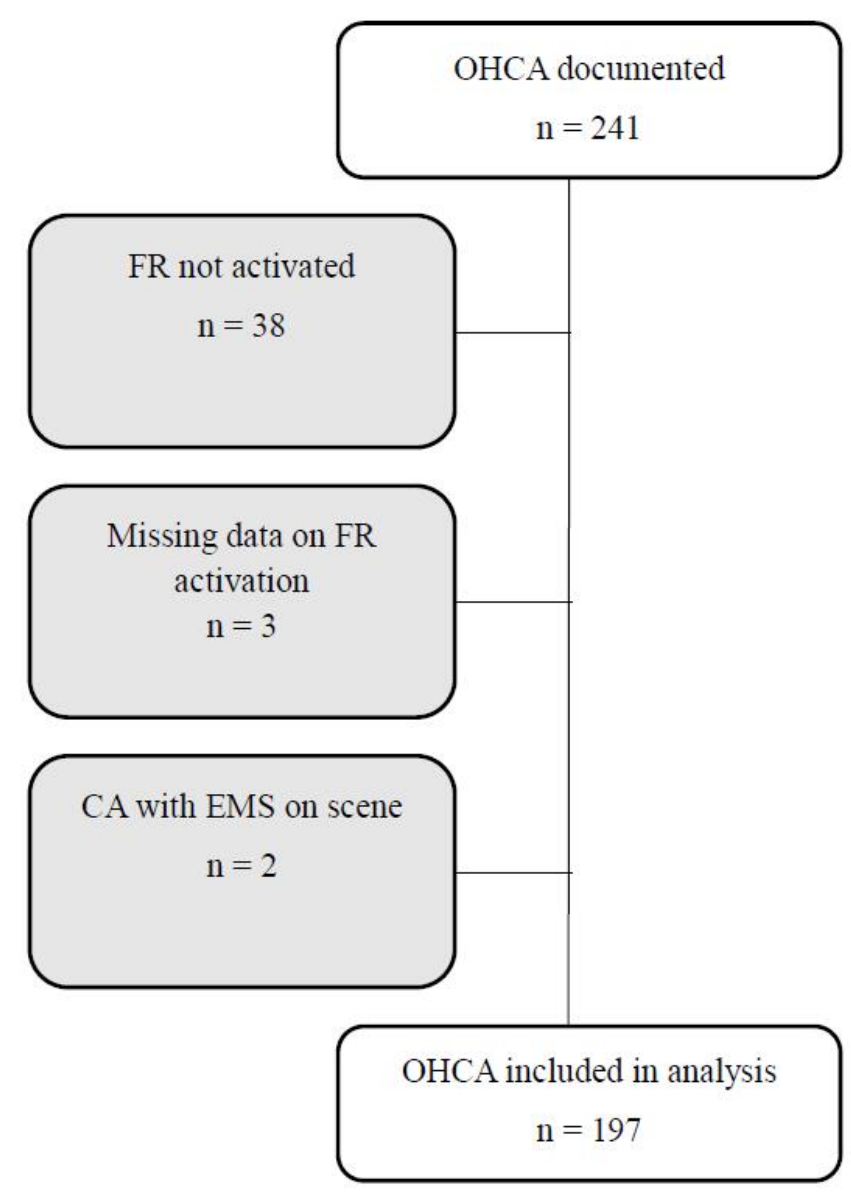

Historical controls

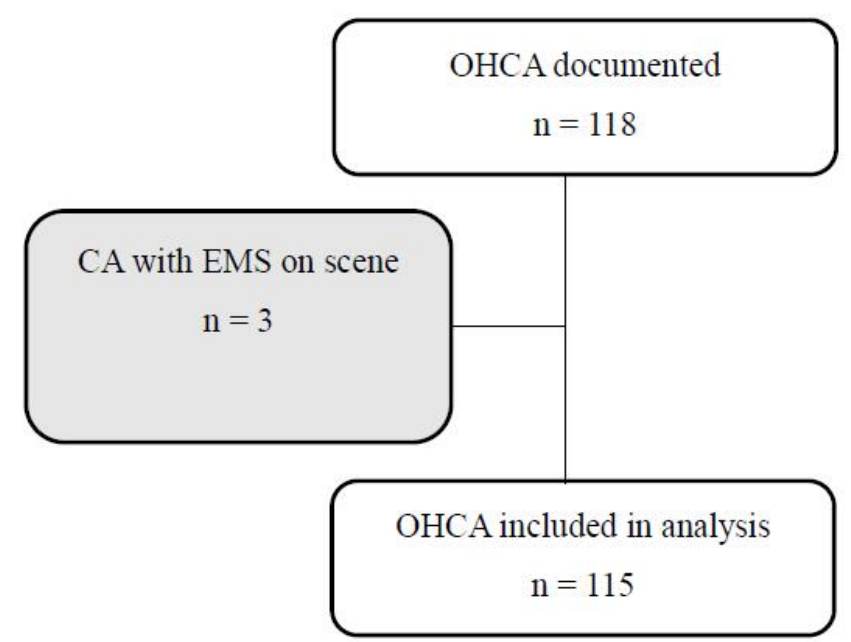

F I G U R E 1. Flowchart of patient inclusion.

Note: OHCA, out-of-hospital cardiac arrest; CA, cardiac arrest; FR, first responders; EMS, emergency medical services.

group. Following variables suggested by previous studies $[11,12,14]$ were added in a multivariable model: intervention (dual dispatch vs historical controls), age: ( $\leq 67$ years (median) vs $\geq 68$ years), gender (female vs male), witnessed status (witnessed vs non-witnessed), bystander CPR (yes vs no), first rhythm (shockable vs non-shockable), etiology (cardiac vs other). Correlation matrix was checked for collinearity which was considered significant if correlations were greater than 0.80 (which was not the case with any of the chosen variables). Associations are presented as odds ratios (ORs) with 95\% confidence intervals (CIs). Results were regarded significant if a two-tailed test yielded a $p$-value equal to or less than 0.05. SPSS software (version 25, SPSS Inc., Chicago, IL, USA) was used for statistical analysis.

\section{Results}

A total of 358 interventions with resuscitation attempts were documented. Three interventions were excluded due to missing data on first responder dispatchment, 38 were excluded because first responders were not activated due to various reasons. Another 5 were excluded because cardiac arrest happened when the EMS was already on scene. The remaining 312 interventions were analyzed in this study (Fig. 1).

Mean age of patients was $66.7 \pm 13.7$ years and $230(73.7 \%)$ were male. Most OHCAs occurred at home, 275 (88.4\%), and most were witnessed, e.g., 233 (76.1\%). In 277 (93.9\%) documented cases the cause of the arrest was cardiac. Bystander CPR was performed in half of the cases (155). Initial rhythm was shockable in 111 cases (36.2\%). Comparisons between historical and intervention groups are reported in Table 1.

There were significant differences in time from call to first shock among groups within intervention group $(p=0.004)$. Patients with ROSC and survivors with CPC $1 / 2$ received the first shock significantly faster compared to all patients together ( $p=0.032$ for both comparisons). In historical group, the time interval to first shock was also significantly shorter in survivors with CPC $1 / 2$ compared to all patients $(p=0.038)$.

The details of the response times (time intervals between call to Medical dispatch center and other time points) are reported and compared separately for all patients, patients with ROSC, and survivors with good neurological outcome (CPC 1 and 2) in both historical and intervention groups in Appendix Table 4.

Characteristics, survival, and neurological outcome of patients with ROSC are presented in Table 2.

In patients with shockable initial rhythm, the number of shocks before the arrival of EMS was significantly higher after first responder implementation $(2.2 \pm 0.8$ in historical group and $2.2 \pm 1.6$ in intervention group; $p<0.001$ ). 
TA B L E 1. Baseline characteristics of patients with OHCA.

\begin{tabular}{|c|c|c|c|c|}
\hline & $\mathrm{N}$ documented (Historical/Intervention) & Historical & Intervention & $p$ \\
\hline Mean age in years (SD) & $114 / 197$ & $64.9(13.7)$ & $67.8(13.6)$ & 0.117 \\
\hline Male gender, $\mathrm{n}(\%)$ & $115 / 197$ & $82(71)$ & $148(75)$ & 0.459 \\
\hline Location of collapse & $115 / 196$ & & & 0.005 \\
\hline At home, n (\%) & & $94(82)$ & $181(92)$ & \\
\hline Other, n (\%) & & $21(18)$ & $15(8)$ & \\
\hline Witnessed collapse, n (\%) & $115 / 191$ & $95(83)$ & $138(72)$ & 0.040 \\
\hline Bystander CPR performed, n (\%) & $113 / 193$ & $54(48)$ & $101(52)$ & 0.443 \\
\hline Initial rhythm shockable, $\mathrm{n}(\%)$ & $76 / 117$ & $37(49)$ & $74(63)$ & 0.337 \\
\hline Cause, n (\%) & $107 / 188$ & & & 0.074 \\
\hline Cardiac & & $104(97)$ & $173(92)$ & \\
\hline Trauma & & $2(2)$ & $3(2)$ & \\
\hline Overdose & & / & $1(0.5)$ & \\
\hline Electrocution & & / & $1(0.5)$ & \\
\hline Asphyxia & & $1(1)$ & $10(5)$ & \\
\hline $\mathrm{N}$ of patients shocked before arrival of EMS, $\mathrm{n}(\%)$ & $59 / 193$ & $7(12)$ & $69(36)$ & $<0.001$ \\
\hline Time from call to arrival of first help ( $\mathrm{min}$ ) & $98 / 195$ & 12 & 8 & $<0.001$ \\
\hline Patients with ROSC & & 11 & 7.5 & 0.002 \\
\hline Survivors with CPC $1 / 2$ & & 10 & 7 & 0.011 \\
\hline Time from call to 1 st shock (min) & $24 / 77$ & 16.5 & 11 & 0.013 \\
\hline Patients with ROSC & & 15 & 7.5 & 0.007 \\
\hline Survivors with CPC $1 / 2$ & & 3.5 & 5 & 0.032 \\
\hline
\end{tabular}

Note: SD, standard deviation; CPR, cardiopulmonary resuscitation; ROSC, return of spontaneous circulation; EMS, Emergency medical services; CPC, Cerebral Performance Category. Percentages in parentheses refer to numbers of documented cases.

A multivariate logistic regression analysis on ROSC is presented in Table 3. The model adequately fitted the data according to Hosmer and Lemeshow test $(p=0.551)$. None of the variables correlated with each other significantly.

The logistic regression model of adjustment confirms the absence of improvement in the ROSC rate after the intervention. In the multivariable logistic regression analysis, the factors associated with improved ROSC were: bystander CPR (adjusted OR 2.0, confidence interval (CI) 1.1-3.6) and shockable first rhythm (adjusted OR 2.4, CI 1.3-4.5).

\section{Discussion}

This observational study showed that the introduction of dual dispatch of voluntary firefighters as local first responders was not associated with increased survival. In our study, reduced response times for patients with OHCA in rural areas as well as a substantial number of OHCA patients resuscitated by first responders were observed.

\subsection{Survival}

Percentage of OHCA patients with initial shockable rhythm did not differ significantly between compared groups in the present study and was similar in other studies [13, 14, 26]. Shorter first responder response times lead to reduced time interval from call to first shock, especially in the group of patients with ROSC. Shorter time intervals to first shock in the two patients that survived without neurological sequelae in the historical controls were resuscitated by bystanders, who were incidentally a health worker and a local firefighter, who was not involved in an organized first responder system and hence stand out from the rest of the population in this study group. Furthermore, a higher number of shocks was delivered by first responders before arrival of EMS. These findings are supported by observations from several studies which also reported that shorter time interval from collapse to initial rhythm analysis more likely resulted in shockable initial rhythm thus increasing chances of ROSC [20, 22, 27]. Unfortunately, we did not observe a statistically significant increase in ROSC, admission to hospital and 30-day survival between compared groups although the hospital admission and 30-day survival rate were similar to other studies [12, 14, 18]. Increased survival to hospital admission and 30day survival were described in metropolitan/suburban areas with dual dispatch system [11, 14, 19]. On the other hand, a twofold increase in favourable neurological outcome in patients with OHCA was found in our study but it did not reach statistical significance. Saner et al. [13] reported about 18 patients with favourable neurological outcome in OHCA patients resuscitated by first responders. These observations could be due to the fact that only patients with shockable rhythm were included in that study, longer time period of 
TA B L E 2. Survival of patients after ROSC.

\begin{tabular}{lcccc} 
& N of responses (Historical/Intervention) & Historical & Intervention & $p$ \\
ROSC, n (\%) & $115 / 196$ & $26(23)$ & $42(21)$ & 0.808 \\
$V F / V T$, n (\%) & $37 / 74$ & $13(35)$ & $21(28)$ & 1.000 \\
Survival to HA, n (\%) & $115 / 196$ & $25(22)$ & $34(17)$ & 0.341 \\
30-day survival, n (\%) & $108 / 191$ & $7(6)$ & $13(7)$ & 0.914 \\
Survival CPC 1/2, n (\%) & $6 / 12$ & $3(50)$ & $11(92)$ & 0.442 \\
\hline Note: ROSC, return of spontaneous circulation; VF/VT, ventricular fibrillation/pulseless ventricular \\
tachycardia; HA, hospital admission; CPC, Cerebral Performance Category.
\end{tabular}

TA B L E 3. Multivariate logistic regression analysis on factors associated with ROSC.

\begin{tabular}{|c|c|c|c|c|c|}
\hline & & No. of cases & ROSC (\%) & Adjusted $^{a}$ OR & $95 \% \mathrm{CI}$ \\
\hline \multirow{2}{*}{ Intervention } & Dual dispatch & 197 & 21.3 & 0.8 & $0.5-1.6$ \\
\hline & Historical control & 115 & 22.6 & & \\
\hline \multirow{2}{*}{ Age } & $\geq 68$ years & 160 & 23.1 & 1.2 & $0.6-2.1$ \\
\hline & $\leq 67$ years & 151 & 20.4 & & \\
\hline \multirow{2}{*}{ Gender } & Female & 82 & 24.4 & 1.4 & $0.7-2.8$ \\
\hline & Male & 230 & 20.9 & & \\
\hline \multirow{2}{*}{ Witnessed status } & Witnessed & 233 & 24.0 & 2.5 & $1.0-6.0$ \\
\hline & Non-witnessed & 73 & 13.7 & & \\
\hline \multirow{2}{*}{ Bystander CPR } & Bystander CPR & 155 & 27.7 & 2.0 & $1.1-3.6$ \\
\hline & No-bystander CPR & 151 & 15.9 & & \\
\hline \multirow{2}{*}{ First rhythm } & Shockable & 111 & 30.6 & 2.4 & $1.3-4.5$ \\
\hline & Non-shockable & 201 & 16.9 & & \\
\hline \multirow{2}{*}{ Etiology } & Cardiac & 277 & 33.3 & 0.3 & $0.1-0.9$ \\
\hline & Other & 18 & 17.6 & & \\
\hline
\end{tabular}

Note: OR, odds ratio; CI, confidence interval. ${ }^{a}$ Adjusted for intervention, age, gender, witnessed status, bystander CPR, first rhythm, etiology.

the study and shorter response times of the firefighters which could contribute to better survival outcome. Despite shorter response times and the fact, that dual dispatch of firefighters provides more skilled people at the scene, we failed to observe increase in ROSC after first responder implementation. This finding is similar to conclusions from Nordberg et al. [12] who also have not found increased survival rates of the OHCA victims after implementation of the dual dispatch with first responders in the rural area of the greater Stockholm area with the population density of $<250 / \mathrm{km}^{2}$. Observations from our study could be due to several factors. One might be that there were more resuscitation attempts in unwitnessed OHCAs as well as in patients of advanced age, both having poorer prognosis $[25,28]$. Delayed advanced life support performed by EMS crew in rural areas also contributes to unfavourable outcome. Nordberg et al. [12] also suggest there could be a breakpoint, after which further reduction in the time interval is of less importance. This observation is supported by survival models where each minute of delay to defibrillate shockable rhythm reduces the probability of survival by $10 \%[5,17,20]$.

\subsection{Time intervals}

Response times of local first responders are significantly shorter compared to EMS response times ( $8 \mathrm{~min}$ vs $12 \mathrm{~min}$, respectively). Similar first responder response times were also reported by other studies [12, 15, 18, 25]. Voluntary firefighters trained as first responders mostly reside in close proximity to the local fire station in the centre of the rural village. After activation, first responders on the way to the OHCA victim usually make a quick stop at the fire station to collect the first aid equipment and an AED. On rare occasions, when a first responder lives closer to the location of the OHCA victim than to the fire station, he or she goes directly to the OHCA victim providing first aid and starts with BLS if necessary, waiting for other first responders to bring the first aid equipment. Such logistics results in shorter response times, which could be further reduced with more optimal dispatch activation. Voluntary firefighters in Slovenia are a part of protection, rescue and relief forces governed by the Administration of the Republic of Slovenia for Civil Protection and Disaster Relief. The request for first responder activation is transferred telephonically from Medical dispatch center via the Regional Emergency Notification Center, which then alerts first responders using a rescue mobile application. 
Consequently, prolonged activation time for first responders was observed in our study compared to Saner et al. [13] (3 min vs $30 \mathrm{sec}$, respectively). Dispatch delay eliminates much of the benefit of the shorter travel time of the first responder [19].

On the other hand, EMS response times were significantly prolonged with the median delay of $8 \mathrm{~min}$ after first responder arrival. Similarly, an increase in EMS response times in rural areas during the first responder project was observed by Moore et al. [25]. This could be due to the fact that more resuscitations were attempted in the remote rural areas with prolonged EMS response times and thus included in the study. Voluntary firefighters as local first responders are trained to perform BLS and AED protocol until arrival of the EMS team, which then takes over the resuscitation effort. Due to different professional backgrounds and limited training as first responders, they are not capable of making crucial decisions. Therefore, they attempt resuscitation of every victim with OHCA regardless of the victim's underlying conditions or whether the collapse was witnessed. Thus, higher numbers of all as well as unwitnessed resuscitations in the intervention group were observed similar to Nordberg et al. [12]. In very few cases, first responders withheld resuscitation attempts due to obvious signs of death (rigor mortis) or in cases where the patient's DNAR order was well known. All these decisions were made after telephone consultation with the on-coming emergency physician. In the study period before the implementation of first responders, EMS arrival times in remote rural areas were prolonged and thus an emergency physician may have recognized resuscitation attempts as futile on arrival. The other reason for prolonged EMS response times could also be less extreme and safer ambulance driving, because when the EMS team is informed that the first responders are activated and on scene, they rely on them to provide BLS and use an AED.

\subsection{Suggestions}

Based on observations from our study the following adjustments to further reduce time from call to arrival of first help and thus improve the survival of OHCA victims are suggested: implementation of a systematic telephone assistance for witnesses to perform chest compressions; immediate activation of the firefighters first responders (not after the EMS); consider an alarm to citizens as lay first responders on a large scale (e.g., via a phone application).

\subsection{Limitations of the study}

Our study has several limitations. First, it is a retrospective study of prospective gathered data which could lead to some bias. When using registry data, results have limitations arising from missing or incorrectly reported variables. Second, the study design makes drawing true conclusions about causality difficult. Third, due to the narrow inclusion criteria the study population is small. Fourth, during the study period the changes in pre- and in-hospital treatment guidelines may have influenced the outcome. Fifth, we did not examine the cost effectiveness of the intervention.

\section{Conclusions}

Introduction of dual dispatch of voluntary firefighters as local first responders in addition to EMS in cases of OHCA significantly shortened response times. However, reduced response times were not associated with better survival outcomes. Nevertheless, based on the results of this study, that is reduced time intervals to first shock and trends towards better neurological outcome in OHCA patients, we encourage implementation of local first responders in rural areas to improve pre-hospital management.

\section{AUTHOR CONTRIBUTIONS}

MS has contributed in the conception and design of the work, acquisition, analysis and interpretation of the data and has substantively revised the drafted work. PJ has contributed substantially to the conception and design of the work, the acquisition and interpretation of the data. VBL contributed to the design of the work, analysis and interpretation of the data and has substantively revised the drafted work. All authors read and approved the final manuscript.

\section{ETHICS APPROVAL AND CONSENT TO PARTICIPATE}

The regional ethics committee of University Medical Center Maribor approved the study and waived the requirement to obtain any informed consent (No. UKC-MB-KME-24/20, 02/010/03-003/01/20). Clinical Trial Registration-URL: http://www.clinicaltrials.gov. Unique identifier: NCT04485390.

\section{ACKNOWLEDGMENT}

We would like to thank all voluntary firefighters who are local first responders for making dual dispatch programme in rural areas possible. We would also like to thank the following colleagues for gathering data from the local EMS protocols and first responder intervention reports and making the sampling possible:

EMS Koroška region: Peter Kordež, EMT; Matej Ploder, $\mathrm{RN}, \mathrm{MSc}$;

EMS Velenje: Aleš Kajtna, MD; Matjaž Andrejc, RN; MSc; EMS Ptuj: Miha Kodela, MD; Matjaž Vaupotič, RN, MSc.

\section{FUNDING}

This research received no external funding.

\section{CONFLICT OF INTEREST}

The authors declare no conflict of interest.

\section{DATA AVAILABILITY}

The data used to support the findings of this study are available from the corresponding author upon request. 


\section{APPENDIX}

See Fig. 2, Table 4.

\section{REFERENCES}

[1] Atwood C, Eisenberg MS, Herlitz J, Rea TD. Incidence of EMS-treated out-of-hospital cardiac arrest in Europe. Resuscitation. 2005; 67: 75-80.

[2] Gräsner JT, Lefering R, Koster RW, Masterson S, Böttiger BW, Herlitz $\mathrm{J}$, et al. EuReCa ONE-27 Nations, ONE Europe, ONE Registry: A prospective one month analysis of out-of-hospital cardiac arrest outcomes in 27 countries in Europe. Resuscitation. 2016; 105: 188-195.

[3] Hasselqvist-Ax I, Riva G, Herlitz J, Rosenqvist M, Hollenberg J, Nordberg P, et al. Early cardiopulmonary resuscitation in out-of-hospital cardiac arrest. New England Journal of Medicine. 2015; 372: 2307-2315.

[4] Malta Hansen C, Kragholm K, Pearson DA, Tyson C, Monk L, Myers $\mathrm{B}$, et al. Association of Bystander and first-Responder Intervention with Survival after out-of-Hospital Cardiac Arrest in North Carolina, 20102013. Journal of the American Medical Association. 2015; 314: 255-264.

[5] Valenzuela TD, Roe DJ, Nichol G, Clark LL, Spaite DW, Hardman RG. Outcomes of rapid defibrillation by security officers after cardiac arrest in casinos. New England Journal of Medicine. 2000; 343: 1206-1209.

[6] Hallstrom AP, Ornato JP, Weisfeldt M, Travers A, Christenson J, McBurnie MA, et al. Public-access defibrillation and survival after outof-hospital cardiac arrest. New England Journal of Medicine. 2004; 351: 637-646.

[7] Capucci A, Aschieri D. Results of early defibrillation program in Piacenza. Minerva Anestesiologica. 2003; 69: 353-356.

[8] Myerburg RJ, Fenster J, Velez M, Rosenberg D, Lai S, Kurlansky P, et al. Impact of community-wide police car deployment of automated external defibrillators on survival from out-of-hospital cardiac arrest. Circulation. 2002; 106: 1058-1064.

[9] Groh WJ, Newman MM, Beal PE, Fineberg NS, Zipes DP. Limited response to cardiac arrest by police equipped with automated external defibrillators: lack of survival benefit in suburban and rural Indianathe police as responder automated defibrillation evaluation (PARADE). Academic Emergency Medicine. 2001; 8: 324-330.

[10] Kooij FO, van Alem AP, Koster RW, de Vos R. Training of police officers as first responders with an automated external defibrillator. Resuscitation. 2004; 63: 33-41.

[11] Nordberg P, Hollenberg J, Rosenqvist M, Herlitz J, Jonsson M, JärnbertPetterson $\mathrm{H}$, et al. The implementation of a dual dispatch system in outof-hospital cardiac arrest is associated with improved short and long term survival. European Heart Journal. 2014; 3: 293-303.

[12] Nordberg P, Jonsson M, Forsberg S, Ringh M, Fredman D, Riva G, et $a l$. The survival benefit of dual dispatch of EMS and fire-fighters in outof-hospital cardiac arrest may differ depending on population density-a prospective cohort study. Resuscitation. 2015; 90: 143-149.

[13] Saner H, Morger C, Eser P, von Planta M. Dual dispatch early defibrillation in out-of-hospital cardiac arrest in a mixed urban-rural population. Resuscitation. 2013; 84: 1197-1202.

[14] Hollenberg J, Riva G, Bohm K, Nordberg P, Larsen R, Herlitz J, et al. Dual dispatch early defibrillation in out-of-hospital cardiac arrest: the SALSApilot. European Heart Journal. 2009; 30: 1781-1789.

[15] Zijlstra JA, Stieglis R, Riedijk F, Smeekes M, van der Worp WE, Koster RW. Local lay rescuers with AEDs, alerted by text messages, contribute to early defibrillation in a Dutch out-of-hospital cardiac arrest dispatch system. Resuscitation. 2014; 85: 1444-1449.

[16] Hansen SM, Brøndum S, Thomas G, Rasmussen SR, Kvist B, Christensen A, et al. Home Care Providers to the Rescue: a Novel first-Responder Programme. PLoS ONE. 2015; 10: e0141352.

[17] White RD, Bunch TJ, Hankins DG. Evolution of a community-wide early defibrillation programme: Experience over 13 years using police/fire personnel and paramedics as responders. Resuscitation. 2005; 65: 279283.

[18] Hasselqvist-Ax I, Nordberg P, Herlitz J, Svensson L, Jonsson M, Lindqvist $\mathrm{J}$, et al. Dispatch of Firefighters and Police Officers in out-ofHospital Cardiac Arrest: a Nationwide Prospective Cohort Trial Using Propensity Score Analysis. Journal of the American Heart Association. 2017; 6: e005873.

[19] van Alem AP, Vrenken RH, de Vos R, Tijssen JGP, Koster RW. Use of automated external defibrillator by first responders in out of hospital cardiac arrest: prospective controlled trial. British Medical Journal. 2003; 327: 1312 .

[20] Waalewijn RA, Nijpels MA, Tijssen JG, Koster RW. Prevention of deterioration of ventricular fibrillation by basic life support during outof-hospital cardiac arrest. Resuscitation. 2002; 54: 31-36.

[21] Hansen SM, Hansen CM, Fordyce CB, Dupre ME, Monk L, Tyson C, et al. Association Between Driving Distance From Nearest Fire Station and Survival of Out-of-Hospital Cardiac Arrest. Journal of the American Heart Association. 2018; 7: e008771

[22] Rajan S, Wissenberg M, Folke F, Hansen SM, Gerds TA, Kragholm $\mathrm{K}$, et al. Association of Bystander Cardiopulmonary Resuscitation and Survival According to Ambulance Response Times after out-of-Hospital Cardiac Arrest. Circulation. 2016; 134: 2095-2104.

[23] Nolan JP, Soar J, Zideman DA, Biarent D, Bossaert LL, Deakin C, et al. European Resuscitation Council Guidelines for Resuscitation 2010 Section 1. Executive summary. Resuscitation. 2010; 81: 1219-1276.

[24] Monsieurs KG, Nolan JP, Bossaert LL, Greif R, Maconochie IK, Nikolaou NI, et al. European Resuscitation Council Guidelines for Resuscitation 2015: Section 1. Executive summary. Resuscitation. 2015; 95: 1-80.

[25] Moore MJ, Hamilton AJ, Cairns KJ, Marshall A, Glover BM, McCann CJ, et al. The Northern Ireland Public Access Defibrillation (NIPAD) study: effectiveness in urban and rural populations. Heart. 2008; 94: 1614-1619.

[26] Zijlstra JA, Koster RW, Blom MT, Lippert FK, Svensson L, Herlitz J, et al. Different defibrillation strategies in survivors after out-of-hospital cardiac arrest. Heart. 2018; 104: 1929-1936.

[27] Renkiewicz GK, Hubble MW, Wesley DR, Dorian PA, Losh MJ, Swain $\mathrm{R}$, et al. Probability of a shockable presenting rhythm as a function of EMS response time. Prehospital Emergency Care. 2014; 18: 224-230.

[28] Beesems SG, Blom MT, van der Pas MHA, Hulleman M, van de Glind EMM, van Munster BC, et al. Comorbidity and favorable neurologic outcome after out-of-hospital cardiac arrest in patients of 70 years and older. Resuscitation. 2015; 94: 33-39.

How to cite this article: Matej Strnad, Pia Jerot, Vesna Borovnik Lesjak. Impact of dual dispatch system implementation on response times and survival outcomes in out-of-hospital cardiac arrest in rural areas. Signa Vitae. 2022;18(1):76-83. doi: $10.22514 /$ sv.2021.134. 


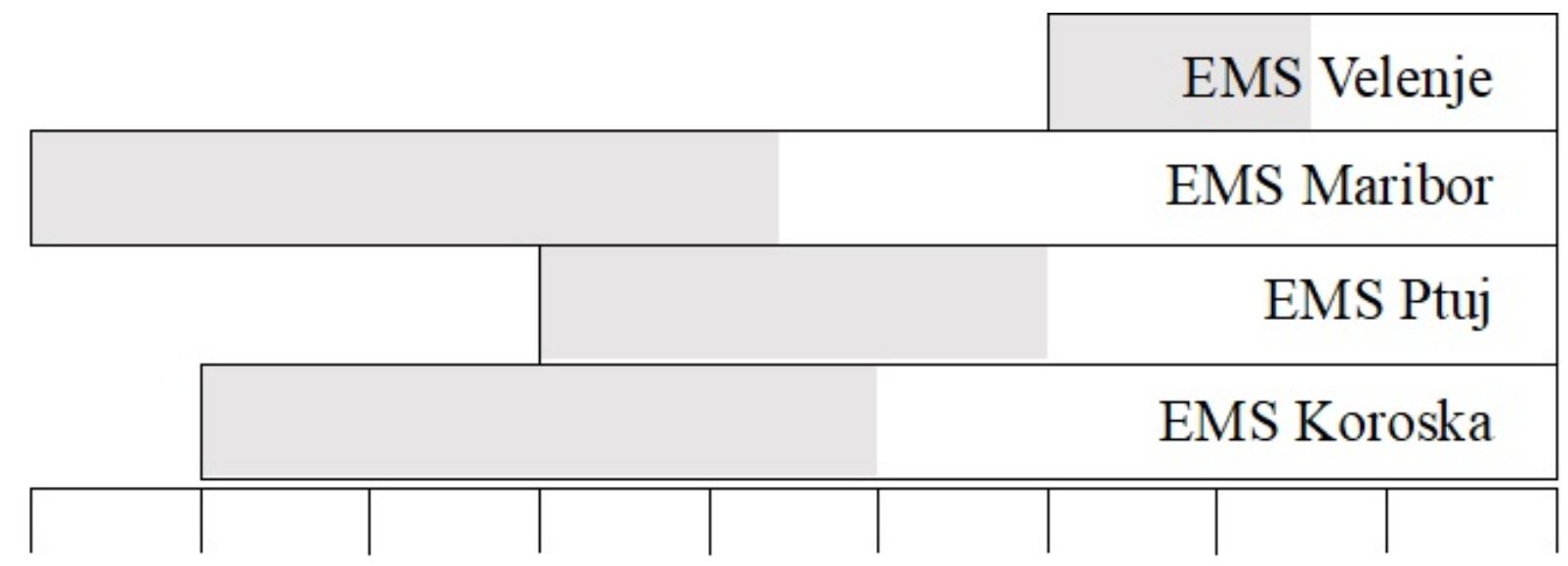

\section{$\begin{array}{llllllllll}2010 & 2011 & 2012 & 2013 & 2014 & 2015 & 2016 & 2017 & 2018 & 2019\end{array}$}

F I G U R E 2. Time frames of OHCA cases' inclusion from different EMS.

Note: EMS, Emergency medical services. Time frames of OHCA cases' inclusion in the historical control group (grey) and intervention group (white) according to implementation of first responders in different catchment areas.

T A B L E 4. Time intervals (median) before and after first responder implementation.

\begin{tabular}{|c|c|c|c|c|c|}
\hline & & $\begin{array}{l}\mathrm{N} \text { of responses (His- } \\
\text { torical/Intervention) }\end{array}$ & Historical & Intervention & $\begin{array}{l}\text { Historical and Intervention } \\
\text { groups comparison, } p \text { value }\end{array}$ \\
\hline \multirow{3}{*}{$\begin{array}{l}\text { Time from call to arrival } \\
\text { of EMS (min) }\end{array}$} & All patients & $111 / 196$ & 13 & 17 & $<0.001$ \\
\hline & Patients with ROSC & & 11 & 16 & 0.283 \\
\hline & Survivors with CPC $1 / 2$ & & 10 & 14 & 0.022 \\
\hline \multirow{3}{*}{$\begin{array}{l}\text { Time from arrival of FR } \\
\text { to arrival of EMS (min) }\end{array}$} & All patients & 195 & l & 8 & l \\
\hline & Patients with ROSC & & l & 8 & / \\
\hline & Survivors with CPC $1 / 2$ & & l & 8 & / \\
\hline \multirow{3}{*}{$\begin{array}{l}\text { Time from call to ROSC } \\
\text { (min) }\end{array}$} & All patients & $24 / 35$ & 26.5 & 28 & 0.713 \\
\hline & Patients with ROSC & & 26.5 & 28 & 0.932 \\
\hline & Survivors with CPC $1 / 2$ & & 16 & 17 & 0.731 \\
\hline \multirow{3}{*}{$\begin{array}{l}\text { Time from call to activa } \\
\text { tion of FR ( } \mathrm{min})\end{array}$} & All patients & 195 & l & 3 & / \\
\hline & Patients with ROSC & & l & 2 & \\
\hline & Survivors with CPC $1 / 2$ & & l & 4 & \\
\hline \multirow{3}{*}{$\begin{array}{l}\text { Time from activation to } \\
\text { arrival of FR (min) }\end{array}$} & All patients & 194 & / & 5 & l \\
\hline & Patients with ROSC & & l & 4.5 & \\
\hline & Survivors with CPC $1 / 2$ & & / & 2 & \\
\hline
\end{tabular}

Note: ROSC, return of spontaneous circulation; CPC, Cerebral Performance Category; FR, first responders. 